Quim. Nova, Vol. 36, No. 8, 1208-1213, 2013

\title{
VALIDAÇÃO DE MÉTODO ANALÍTICO LIVRE DE ACETONITRILA PARA ANÁLISE DE MICROCISTINAS POR CROMATOGRAFIA LÍQUIDA DE ALTA EFICIÊNCIA
}

\author{
Sérvio Túlio Alves Cassini*, Paulo Wagner Pereira Antunes e Regina Keller \\ Departamento de Engenharia Ambiental, Universidade Federal do Espírito Santo, 29075-910 Vitória - ES, Brasil
}

Recebido em 8/11/12; aceito em 25/4/13; publicado na web em 13/6/13

\begin{abstract}
VALIDATION OF THE ACETONITRILE-FREE ANALYTICAL METHOD FOR MICROCYSTIN ANALYSIS BY HIGH PERFORMANCE LIQUID CHROMATOGRAPHY. Blooms of cyanobacteria represent a public health risk due to their cyanotoxins such as microcystins. Liquid chromatography techniques to separate and quantify microcystins invariably use acetonitrile as the organic component of the mobile phase. The price and availability of acetonitrile together with its elevated toxicity encourage the validation of acetonitrile-free methods of microcystin analysis. In this work, methanol was employed as the organic solvent of the mobile phase and the validation method was performed with different environmental water samples. The method showed limits of detection between 0.17 and $0.25 \mu \mathrm{g} / \mathrm{L}$ and of quantification between 0.55 and $0.82 \mu \mathrm{g} / \mathrm{L}$ for the microcystin variants: -RR, -YR, -LR, -LA.
\end{abstract}

Keywords: microcystin; acetonitrile; methanol.

\section{INTRODUÇÃO}

A modificação de ambientes aquáticos pelo acúmulo de nutrientes produzidos nos diferentes processos antropogênicos é a principal causa de eutrofização em rios e lagos em todo o mundo. A eutrofização é apontada como sendo um dos principais fatores responsável pelo aumento das florações de cianobactérias. Essas florações promovem a deterioração da qualidade da água e constituem um sério risco à saúde pública. ${ }^{1,2}$ As cianobactérias produzem metabólitos tóxicos denominados cianotoxinas que podem ser letais para os animais selvagens, domésticos e seres humanos. As cianotoxinas podem causar graves irritações na pele, além de efeitos neurotóxicos e hepatóxicos. ${ }^{3,4}$

As florações de cianobactérias nem sempre são compostas de espécies tóxicas, entretanto 50 a $70 \%$ das florações apresentam toxicidade. A ocorrência de florações tóxicas não é apenas um fenômeno local, regional ou especifico de um só país, mas de proporções globais. ${ }^{5-9}$ As ocorrências de florações e o controle de suas toxinas em águas de abastecimento descrevem representantes do gênero Microcystis e a cianotoxina microcistina como sendo os mais comuns em florações no Brasil e no mundo. ${ }^{10-13}$ Portanto, nos estudos com florações, a microcistina é utilizada como padrão para detectar e quantificar a presença de cianotoxinas. Seguindo as recomendações da Organização Mundial da Saúde (OMS), a legislação brasileira estabeleceu o valor de 1,0 $\mu \mathrm{g} / \mathrm{L}$ como sendo a concentração máxima de microcistina permitida em águas destinadas ao abastecimento público. ${ }^{14-16}$

As microcistinas são peptídeos cíclicos compostos por sete resíduos de aminoácidos (Figura 1). A estrutura cíclica proporciona uma extrema estabilidade em água e tolerância a mudanças radicais de $\mathrm{pH}$ e temperatura, mantendo a toxicidade das microcistinas mesmo após a fervura. ${ }^{3}$ Nas posições 2 e 4 da estrutura são encontrados resíduos de L-aminoácidos responsáveis pela nomenclatura das diferentes variantes de microcistinas. ${ }^{17}$ A separação e quantificação destas variantes podem ser realizadas por técnicas analíticas de cromatografia líquida de alta eficiência (CLAE) baseada na diferente hidrofobicidade destes resíduos de L-aminoácidos. Invariavelmente, as técnicas cromatográficas de análises de microcistinas utilizam fase reversa de separação (CLAE-FR) e o solvente acetonitrila (ACN) como componente orgânico da fase móvel. ${ }^{18-20}$

\footnotetext{
*e-mail: scassini@terra.com.br
}

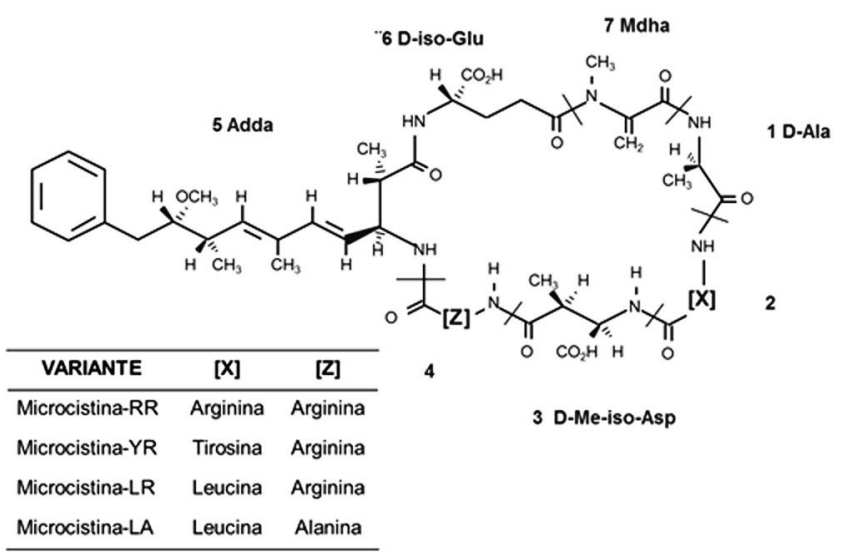

Figura 1. Estrutura geral de microcistinas e as principais variantes detectadas em florações tóxicas. [X] e [Z] representa resíduos de L-aminoácidos responsáveis pela nomenclatura das diferentes variantes de microcistinas

A ACN é um subproduto da produção de fibras e resinas de acrílico, produtos de uso predominante na fabricação de automóveis, eletrodomésticos e eletrônicos. No final de 2008, a disponibilidade de ACN diminuiu em todo o mundo por diferentes razões, principalmente devido à crise econômica global que reduziu a demanda por produtos a base destas fibras e resinas. O preço da ACN chegou a custar cinco vezes mais na Europa, devido uma redução de $80 \%$ na sua oferta. ${ }^{21}$ Embora seja difícil uma nova combinação de fatores capaz de promover uma alta redução no fornecimento de ACN como ocorreu em 2008, o volume deste solvente é gerado devido a processos industriais que estão sujeitos a crises e retrações mundiais. Além disso, outra preocupação em relação ao uso de ACN é o seu alto potencial toxicológico. A ACN é prontamente absorvida quando em contato com trato gastrointestinal, pele e pulmões, espalhando-se rapidamente por todo corpo. A intoxicação por ACN pode causar graves efeitos sistêmicos devido a sua degradação em cianeto, um potente inibidor da cadeia transportadora de elétrons. ${ }^{22}$

Nas análises cromatográficas, diferentes modificadores podem ser utilizados em alternativa ao uso da ACN. A seletividade da técnica de CLAE-FR é influenciada pelo tipo de modificador orgânico da fase móvel, isto porque o mecanismo de retenção do analito na fase 
estacionária depende do tipo de modificador utilizado. ${ }^{23}$ Além do metanol $(\mathrm{MeOH})$, outros solventes tais como etanol, isopropanol e acetona podem ser utilizados como modificadores orgânicos. Entretanto a alta absorção na região UV-VIS e a necessidade de pressões mais elevadas dificultam a ampla utilização destes solventes. ${ }^{24} \mathrm{O}$ etanol merece destaque por se tratar de um solvente muito menos tóxico quando comparado à aqueles mais utilizados tais como metanol e acetonitrila. ${ }^{25}$ Porém, o aumento da viscosidade quando dissolvidos em água dificulta a aplicação do etanol e metanol, principalmente em sistemas cromatográficos mais antigos, os quais apresentam sistemas de bombas mais sensíveis à variação de pressão. ${ }^{26}$

Nas técnicas de CLAE-FR, o menor caráter hidrofóbico do $\mathrm{MeOH}$ quando comparado à $\mathrm{ACN}$ exige um aumento de sua concentração na fase móvel. Além disso, a sua menor viscosidade em soluções aquosas é responsável por um aumento na pressão do sistema. Porém, apesar de pequenas alterações com relação à linha de base e no tempo de retenção dos analitos avaliados, a utilização do $\mathrm{MeOH}$ como modificador orgânico nas técnicas de CLAE-FR demonstra eficiência de separação e seletividade semelhante às técnicas que utilizam $\mathrm{ACN} .{ }^{23}$ $\mathrm{O} \mathrm{MeOH}$ também apresenta risco potencial para a saúde humana, desenvolvendo efeitos colaterais semelhantes à $\mathrm{ACN}$, porém os níveis de exposição ocupacional e concentração letal é cerca de duas vezes maior (1,5 a 2,0 g/m $\mathrm{m}^{3} \mathrm{~h}$ ). Os efeitos crônicos de inalação também são menores. $\mathrm{O}$ valor limite de exposição ocupacional recomendado para turnos de 8 horas é $0,26 \mathrm{~g} / \mathrm{m}^{3}$ de $\mathrm{MeOH}$ dissolvido no ar. Este valor é mais do que três vezes a concentração media de ACN recomendada para se evitar qualquer efeito tóxico. ${ }^{27}$

Nas análises de microcistinas, o $\mathrm{MeOH}$ é o principal solvente utilizado na extração da toxina a partir de células de cianobactérias. O desenvolvimento de técnicas cromatográficas de separação de microcistinas utilizando o $\mathrm{MeOH}$ como composto orgânico da fase móvel, além de reduzir custos e toxicidade em relação ao uso de ACN, ainda diminui o grau de limpeza necessária para remoção de possíveis contaminantes presentes na amostra. ${ }^{21}$ Portanto, o objetivo deste estudo foi padronizar e validar um método de separação e quantificação de microcistinas utilizando a cromatografia líquida de alta eficiência e metanol como componente orgânico da fase móvel em alternativa ao uso da acetonitrila.

\section{EXPERIMENTAL}

\section{Reagentes}

O metanol grau CLAE utilizado foi da marca Tedia (Tedia Company, Fairfield, USA). O ácido Trifluoracético (TFA) foi adquirido na Merck (Darmstadt, Germany). A água foi purificada no sistema de purificação da Millipore (Bedford, USA). Os cartuchos de extração em fase sólida (SPE) da marca Chromabond ${ }^{\circledR}$ C18ec (6 mL, 500 mg) foram obtidos da Macherey-Nagel (Duren, Germany). As variantes de microcistinas utilizadas (-RR, -YR, -LR e -LA) foram padrões analíticos para análises ambientais, da marca Sigma (St.Louis, USA). Todos os outros reagentes utilizados foram de grau analítico.

\section{Preparo de amostras}

A fim de simular a contaminação com microcistinas, amostras ambientais de águas foram fortificadas nas concentrações finais de 0,$8 ; 1,0$ e 2,0 $\mu \mathrm{g} / \mathrm{L}$. Estas amostras foram coletadas no lago de Duas Bocas e nos rios Jucu e Santa Maria, principais mananciais utilizados na captação de água para o abastecimento público da região metropolitana de Vitória-ES. Após a fortificação, volumes de $500 \mathrm{~mL}$ das amostras foram submetidos à extração em fase sólida por adsorção em cartuchos C18, conforme a norma ISO 20179:2005. Em seguida, o material adsorvido no cartucho foi eluído com 6,0 mL de solução de MeOH:H ${ }_{2} \mathrm{O}: T F A(89,9: 10: 0,1$ v/v) e submetido à evaporação à temperatura ambiente. $\mathrm{O}$ material foi então ressuspenso com $0,5 \mathrm{~mL}$ de solução de $\mathrm{MeOH}: \mathrm{H}_{2} \mathrm{O}(80: 20$ v/v) para as análises cromatográficas.

\section{Análises cromatográficas}

A análise das amostras foi realizada em um sistema de cromatografia da série Shimadzu CBM-20A, equipado com um desgaseificador de solventes DGU-20AS, uma bomba quaternária de gradiente LC-20AT, um injetor automático de amostras SIL-20AHT e um detector de arranjo de diodos SPD-M20A. A coluna cromatográfica utilizada neste estudo foi a coluna analítica Kinetex ${ }^{\mathrm{TM}} \mathrm{C} 18(100 \mathrm{x}$ 2,1 mm, 2,6 $\mu \mathrm{m}, 100 \mathrm{~nm}$ ). O comprimento de onda de detecção foi de $238 \mathrm{~nm}$. O volume de injeção para as análises foi de $50 \mu \mathrm{L}$ da amostra e a vazão da fase móvel foi de $0,250 \mathrm{~mL} / \mathrm{min}$. Em todas as análises, a coluna permaneceu acondicionada à temperatura constante de $37{ }^{\circ} \mathrm{C}$ utilizando um módulo aquecedor de coluna da Shimadzu.

A solução de $\mathrm{H}_{2} \mathrm{O}$ :MeOH:TFA (69,9:30:0,1 v/v) foi utilizada como fase móvel A, enquanto a fase B foi composta por metanol $100 \%$. O vazão utilizada nas análises foi de $0,250 \mathrm{~mL} / \mathrm{min}$. O perfil do gradiente iniciou-se com $100 \%$ da fase móvel A e foi executado de forma isocrática no intervalo de 0 a $1 \mathrm{~min}$. Entre 1 e 3 minutos aplicou-se um gradiente linear de metanol variando a porcentagem da fase móvel B de 0 a 20\%. Entre 3 e 8 minutos o gradiente linear variou de 20 a $40 \%$. Em seguida, o gradiente linear de 40 a $62 \%$ foi aplicado no intervalo de 8 e 25 minutos. Entre 25 e 25,5 minutos, o gradiente retornou à condição inicial e manteve-se até o re-equilíbirio da coluna. Todas as soluções preparadas para compor as fases móveis foram previamente filtradas e desgaseificadas por 15 minutos em banho de ultrassom (Limpsonic ${ }^{\circledR}$, Brasil) antes de serem utilizadas.

\section{Validação do método}

As variantes de microcistinas (Mcyst-RR, -YR, -LR e -LA) foram dissolvidas em solução de $\mathrm{MeOH} 20 \%$ e a solução padrão de $10 \mu \mathrm{g} /$ $\mathrm{mL}$ foi estocada a $-20{ }^{\circ} \mathrm{C}$. A curva analítica foi obtida pela injeção de soluções padrões com concentrações entre 0,2 e $2,0 \mu \mathrm{g} / \mathrm{mL}$. Os dados foram processados utilizando o software LCSolutions (versão 2.1). O coeficiente de correlação, a inclinação e o intercepto da curva padrão, bem como a tabela de análise de variância e os respectivos erros padrões foram calculados para verificar a validação do modelo linear da equação utilizada para padronizar o método de quantificação. Os parâmetros determinados para a validação do método foram: Especificidade, Linearidade, Limite de detecção (LOD), Limite de quantificação (LOQ), Recuperação, Precisão e Robustez. Os parâmetros de desempenho do método foram determinados de acordo com a Resolução no 899/2003 da ANVISA - Agência Nacional de Vigilância Sanitária. ${ }^{28}$

\section{RESULTADOS E DISCUSSÃO}

Testes de eluição foram realizados para definir a melhor condição analítica para separação e quantificação das diferentes variantes de microcistina (Mcyst). Na Figura 2 está representado o cromatograma referente à separação de uma amostra contendo as variantes Mcyst-RR, -YR, -LR e LA na concentração final de 1,0 $\mu \mathrm{g} / \mathrm{mL}$. O método permitiu a eluição distinta de cada variante com o tempo de retenção variando entre 22 e 30 minutos (Mcyst-RR: 22,3 min; Mcyst-YR: 24,3 min; Mcyst-LR: 25,7 min e Mcyst-LA: 29,7 min). A alta definição e elevada intensidade dos picos referentes às variantes demonstram a eficiência e seletividade do método. Apesar do elevado tempo de análise, o método mostra-se favorável, pois com a vazão de 
0,250 $\mathrm{mL} / \mathrm{min}$ a análise requer uma baixa quantidade de solvente. Durante a padronização, tentou-se a redução do tempo de eluição principalmente nos primeiros 20 minutos, porém o aumento da vazão provocou a superposição dos picos, impedindo a separação das variantes. Além disso, nas análises das amostras foi observada a eluição de interferentes nos primeiros 20 minutos.

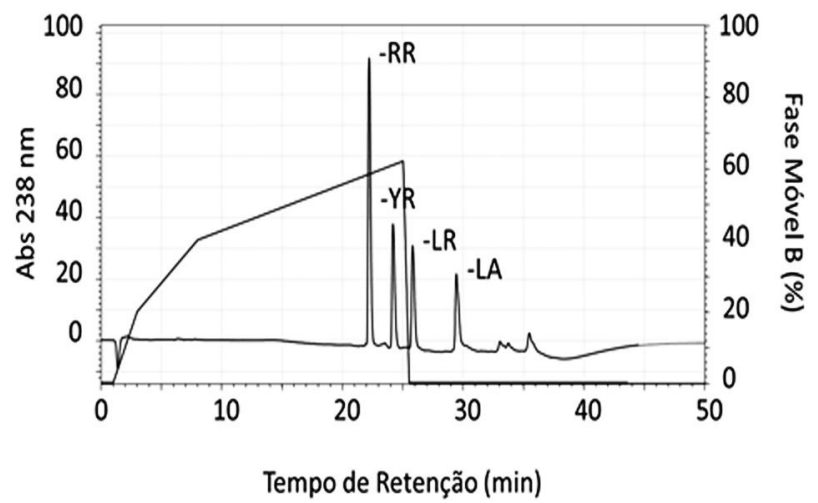

Figura 2. Perfil cromatográfico da amostra padrão contendo as variantes Mcyst-RR (22,3 min), Mcyst-YR (24,3 min), Mcyst-LR (25,7 min) e Mcyst-LA (29,7 min), na concentração final de 1,0 $\mu \mathrm{g} / \mathrm{mL}$. As soluções de $\mathrm{H}_{2} \mathrm{O}: \mathrm{MeOH}: \mathrm{TFA}(69,9: 30: 0,1 \mathrm{v} / \mathrm{v})$ e MeOH 100\% foram utilizadas como fase móvel A e B, respectivamente. A taxa de vazão foi de $0,250 \mathrm{~mL} / \mathrm{min}$ e a detecção foi realizada no comprimento de onda de $238 \mathrm{~nm}$

A especificidade de um método é a capacidade de medir exatamente um composto em presença de outros componentes tais como impurezas, produtos de degradação e componentes da matriz. ${ }^{28}$ Sendo assim, o teste de especificidade foi realizado comparando-se uma amostra ambiental de água fortificada e não fortificada com uma amostra padrão contendo as variantes de Mcyst-RR, -YR, -LR e -LA dissolvidas em solução de $\mathrm{H}_{2} \mathrm{O}: \mathrm{MeOH}(80: 20 \mathrm{v} / \mathrm{v})$, na concentração final de $1,0 \mu \mathrm{g} / \mathrm{L}$. A amostra ambiental foi fortificada com o padrão de Mcyst-LR na concentração final de 1,0 $\mu \mathrm{g} / \mathrm{L}$. Em seguida, as amostras ambientais foram concentradas e injetadas no cromatógrafo a líquido e seus perfis de eluição foram comparados com o perfil obtido com a amostra contendo as variantes de Mcyst. A sobreposição dos perfis cromatográficos (Figura 3) demonstra a ausência de interferência de compostos da matriz capaz de prejudicar a determinação das variantes. Quando se compara os resultados de separação da amostra fortificada com a amostra do padrão de Mcyst observa-se a capacidade da técnica em medir a resposta analítica das variantes de Mcyst na presença de impurezas presentes na amostra ambiental de água, demonstrando a especificidade do método.

A linearidade da resposta de detecção das variantes de Mcyst pela técnica cromatográfica foi avaliada pela relação entre a concentração

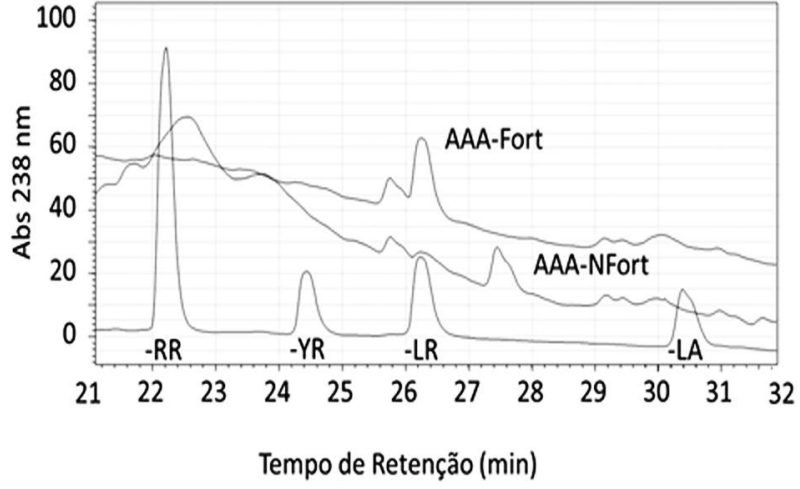

Figura 3. Sobreposição dos perfis cromatográficos referentes ao padrão de Mcyst-LR em solução de $\mathrm{H}_{2} \mathrm{O}: \mathrm{MeOH}(80: 20 \mathrm{v} / \mathrm{v})$, amostra ambiental de água não fortificada (AAA-NFort) e fortificada (AAA-Fort) com o padrão de Mcyst-LR. As soluções de $\mathrm{H}_{2} \mathrm{O}: \mathrm{MeOH}$ :TFA (69,9:30:0,1 v/v) e MeOH 100\% foram utilizadas como fase móvel A e B, respectivamente. A taxa de vazão foi de $0,250 \mathrm{~mL} / \mathrm{min}$ e a detecção foi realizada no comprimento de onda de $238 \mathrm{~nm}$

injetada e a área do pico de resposta. A faixa de concentração avaliada foi de 0,20 a $2,0 \mu \mathrm{g} / \mathrm{mL}$, equivalente a 20 e $200 \%$ do valor máximo de microcistina $(1,0 \mu \mathrm{g} / \mathrm{mL})$ permitido pela legislação brasileira em amostras de águas para abastecimento publico. A linearidade foi avaliada por sete repetições de cada nível de concentração e a curva analítica foi estabelecida para cada variante de Mcyst (Tabela 1).

Os resultados demonstraram a capacidade da técnica em correlacionar diretamente os valores da concentração de Mcyst na amostra com a área do pico de resposta para todas as variantes avaliadas. A resposta linear da técnica foi satisfatória, apresentando coeficientes de correlação (r) superiores a 0,99. Valores de coeficientes semelhantes também foram observados em diferentes estudos para determinação de Mcyst utilizando técnicas de CLAE com ACN como modificador orgânico. Em separações associadas ao sistema de detecção por espectrometria de massas tipo MALDI-TOF (Matrix Assisted Laser Desorption/Ionization-Time of Flight) observou-se a linearidade no intervalo de concentração de Mcyst-LR de 0,03 a 2,00 $\mu \mathrm{g} / \mathrm{mL}$, com o coeficiente de correlação de $0,9996 .{ }^{20}$ Estudo de separação da variante Mcyst-LR associada ao sistema de detecção por espectrofotometria UV/Visível apresentou linearidade no intervalo de 0,05 a 5,00 $\mu \mathrm{g} / \mathrm{mL}$, com valor de (r) igual a $0,9926 .{ }^{29}$ Já quando a separação de cinco variantes de Mcyst (-RR, -LR, -LY, -LW e -LF) foi associada ao sistema de detecção por arranjos de diodos, os valores de (r) variaram entre 0,9630 e 1,000, para o intervalo de linearidade de 0 a $10,00 \mu \mathrm{g} / \mathrm{mL} .^{30}$ A ANVISA recomenda um coeficiente de correlação igual a 0,99 e o Instituto Nacional de Metrologia, Normalização e Qualidade Industrial (INMETRO) um valor acima de $0,90 .^{30}$

Tabela 1. Parâmetros de linearidade da curva analítica $(y=\alpha x+\beta)$ obtida para as variantes de microcistina

\begin{tabular}{|c|c|c|c|c|}
\hline Concentração $(\mu \mathrm{g} / \mathrm{mL})$ & $\begin{array}{l}\text { Área média } \\
\text { Mcyst-RR }\end{array}$ & $\begin{array}{l}\text { Área média } \\
\text { Mcyst-YR }\end{array}$ & $\begin{array}{l}\text { Área média } \\
\text { Mcyst-LR }\end{array}$ & $\begin{array}{l}\text { Área média } \\
\text { Mcyst-LA }\end{array}$ \\
\hline 0,2 & 141299 & 57087 & 56739 & 49485 \\
\hline 0,4 & 469009 & 214089 & 291727 & 194825 \\
\hline 0,8 & 979914 & 453304 & 516492 & 405162 \\
\hline 1,0 & 1468769 & 670218 & 758850 & 552474 \\
\hline 2,0 & 3136349 & 1620747 & 1641211 & 984799 \\
\hline Intercepto com eixo y $(\beta)$ & -231785 & -165041 & -111312 & -14140 \\
\hline Inclinação da reta $(\alpha)$ & 1671424 & 872875 & 868541 & 513055 \\
\hline Coeficiente de correlação (r) & 0,9960 & 0,9920 & 0,9950 & 0,9900 \\
\hline
\end{tabular}


A robustez de uma técnica analítica é a medida de sua capacidade em resistir a pequenas e aleatórias variações dos parâmetros analíticos otimizados. Em CLAE, a robustez pode ser avaliada, por exemplo, variando-se o $\mathrm{pH}$ da fase móvel, a temperatura de acondicionamento da coluna ou vazão da fase móvel. Na validação da técnica em questão, o teste de robustez foi avaliado variando-se as temperaturas de acondicionamento da coluna e as concentrações de ácido trifluoracético (TFA) na fase móvel aquosa. As temperaturas avaliadas foram de 31,34 e $37^{\circ} \mathrm{C}$, já as concentrações de TFA adicionadas à fase aquosa foram $0 ; 0,01 ; 0,05$ e $0,10 \%(\mathrm{v} / \mathrm{v})$. As demais condições otimizadas foram fixadas (vazão $0,250 \mathrm{~mL} / \mathrm{min}$ e a fase móvel orgânica MeOH 100\%).

Os resultados demonstraram robustez da técnica no intervalo de temperatura avaliada (Figura 4). Em relação à variação da temperatura de acondicionamento das colunas, as resoluções cromatográficas entre as variantes de Mcyst não apresentaram diferenças, apenas pequenas variações no tempo de retenção. A temperatura de $37^{\circ} \mathrm{C}$ apresentou o menor tempo de retenção e foi utilizada para o acondicionamento da coluna nas demais análises de validação. Além do menor tempo de retenção, a temperatura de $37^{\circ} \mathrm{C}$ permitiu a execução das análises com menor pressão no sistema cromatográfico, já que a viscosidade da fase móvel decresce exponencialmente em função da temperatura. ${ }^{31}$ Os resultados dos testes de variação do $\mathrm{pH}$ da fase móvel aquosa demonstraram a robustez do técnica com concentrações de TFA de 0,01 a $0,10 \%$ (Figura 4). A resolução cromatográfica obtida com a fase móvel contendo TFA $0,10 \%$ foi definida como condição ótima para os demais ensaios cromatográficos.

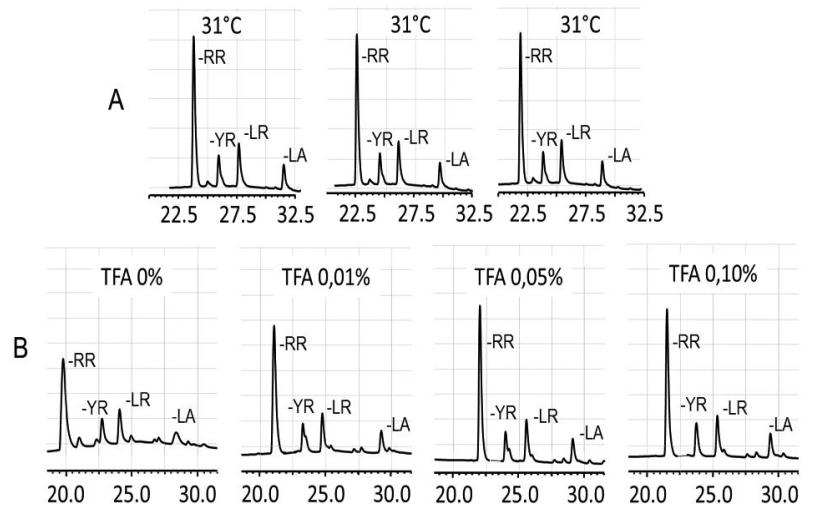

Figura 4. Perfis cromatográficos referentes ao padrão de Mcyst-RR, Mcyst-YR, Mcyst-LR e Mcyst-LA em solução de $\mathrm{H}_{2} \mathrm{O}: \mathrm{MeOH}(80: 20 \mathrm{v} / \mathrm{v})$, na concentração de $1,0 \mu \mathrm{g} / \mathrm{mL}$. (A) Análises realizadas com diferentes temperaturas de acondicionamento da coluna $\left(31,34\right.$ e $\left.37^{\circ} \mathrm{C}\right)$. (B) Análises realizadas diferentes concentrações de ácido trifluoracético adicionado à fase móvel aquosa $(0$; 0,$01 ; 0,05$ e $0,10 \% \mathrm{v} / \mathrm{v}$ )

A capacidade do método de detectar pequenas concentrações é expressa pelo Limite de detecção (LOD) e Limite de Quantificação (LOQ). O método é considerado sensível quando pequenas variações nas concentrações expressam respostas analíticas significativas. Para a determinação de LOD e LOQ do método, soluções foram preparadas com concentrações de 0,$80 ; 1,0 ; 1,5$ e $2,0 \mu \mathrm{g} / \mathrm{L}$ das variantes de Mcyst-RR, -YR, -LR e -LA. O LOD e LOQ foram calculados a partir dos valores de desvio padrão, do intercepto com o eixo y e o valor médio da inclinação da curva analítica. Estes valores foram calculados utilizando as curvas analíticas obtidas com três repetições para cada nível de concentração. De acordo com os resultados obtidos, o método é capaz de detectar com eficiência as variantes avaliadas a partir da concentração de $0,17 \mu \mathrm{g} / \mathrm{L}$ (LOD), valor de LOD observado para Mcyst-RR. Os limites de quantificação (LOQ) variaram entre
0,55 (Mcyst-RR) e 0,82 $\mu \mathrm{g} / \mathrm{L}$ (Mcyst-YR) (Tabela 2). Sistemas de separação de Mcyst utilizando técnicas de CLAE-FR e ACN como modificador orgânico, quando associado ao sistema de detecção por espectrometria de massas tipo MALDI-TOF (Matrix Assisted Laser Desorption/Ionization-Time of Flight), apresentaram valores de LOD e LOQ de 0,05 e $0,15 \mu \mathrm{g} / \mathrm{mL}$, para Mcyst-LR. ${ }^{20}$ Quando o sistema de detecção foi do tipo ESI-MS (Electrospray Ionization Mass spectrometry), a capacidade de detecção foi ainda maior, com o LOD de $0,002 \mu \mathrm{g} / \mathrm{mL} .{ }^{32}$ Embora as técnicas de CLAE-FR utilizando ACN demonstrem menores valores de LOD e LOQ, a técnica utilizando o metanol, aqui validada, mostra-se satisfatória, pois os valores encontrados para LOD e LOQ do método são menores que o valor máximo permitido pela legislação brasileira para detecção de Mcyst em amostras de águas para abastecimento público.

Tabela 2. Limite de detecção (LOD) e limite de quantificação (LOQ) na determinação das variantes de Mcyst-RR, Mcyst-YR, Mcyst-LR e Mcyst-LA, empregando CLAE-FR associado ao detector tipo PDA (Photo Diodo Array)

\begin{tabular}{ccc}
\hline & \multicolumn{2}{c}{ Detector PDA } \\
\cline { 2 - 3 } & $\mathrm{LOD}(\mu \mathrm{g} / \mathrm{L})$ & $\mathrm{LOQ}(\mu \mathrm{g} / \mathrm{L})$ \\
\hline Mcyst-RR & 0,17 & 0,55 \\
Mcyst-YR & 0,25 & 0,82 \\
Mcyst-LR & 0,21 & 0,70 \\
Mcyst-LA & 0,22 & 0,75 \\
\hline
\end{tabular}

A precisão e a acurácia determina o erro de uma medição analítica e são critérios primários para se avaliar a eficiência da técnica analítica. A precisão define a repetibilidade da técnica e determina o desvio da análise em relação ao resultado obtido. Os testes de repetibilidade do método foram expressos por meio da estimativa do desvio padrão relativos (DPR) de sete replicatas de amostras de diferentes corpos d'água da região metropolitana de Vitória-ES (Tabela 3). Volumes de $500 \mathrm{~mL}$ da amostra de água foram coletados e fortificados com Mcyst-LR, na concentração final de 1,0 $\mu \mathrm{g} / \mathrm{L}$. Em seguida, foram concentrados em cartuchos de extração tipo SPE e analisados no sistema de cromatografia líquida. Para técnicas analíticas de separação e quantificação em amostras com pequenas concentrações de impurezas, o DPR de até $20 \%$ é aceitável. ${ }^{33}$ No entanto, o guia de validação da ANVISA não permite que valores de DPR sejam superiores a $5 \% .{ }^{28} \mathrm{O}$ valor de DPR igual a 3,2\% encontrado é inferior aos valores aceitos, indicando a precisão do método.

Neste trabalho, a precisão do método também foi avaliada por experimentos de recuperação. Isto porque para separar e quantificar variantes de Mcyst em amostras ambientais de água, pela técnica cromatográfica, é necessário uma preparação previa das amostras. Esta preparação visa clarificar e concentrar a amostra antes de ser injetada no cromatógrafo. ${ }^{34}$ Devido à presença desta etapa prévia para o processo de validação, passa a ser necessário calcular o fator de recuperação para diferentes concentrações de Mcyst. O teste de recuperação foi realizado pela adição da variante de Mcyst-LR em sete amostras de água. As concentrações utilizadas foram de 0,80 ; 1,0 e $2,0 \mu \mathrm{g} / \mathrm{L}$. Estes valores representam, respectivamente, o ponto médio da curva analítica, o valor máximo permitido pela legislação brasileira e o limite superior da curva. A percentagem de recuperação foi observada entre 98,18 e 106,13\% (Tabela 4).

A precisão de técnicas analíticas que utilizam tratamento prévio das amostras deve ser avaliada pela percentagem de recuperação, através de ensaios com amostras fortificadas. Embora seja desejável atingir níveis de recuperação próximos de $100 \%$, maximizando a sensibilidade da técnica, é provável que níveis de recuperação acima de $70 \%$ não comprometam a integridade da técnica. Um intervalo 
Tabela 3. Valores de recuperação, média e coeficiente de variação $(\mathrm{CV})$ para diferentes amostras de água fortificadas com microcistina-LR na concentração final de $1,0 \mu \mathrm{g} / \mathrm{L}$

\begin{tabular}{|c|c|c|c|c|c|}
\hline Repetição & Amostra & Mcyst-LR $(\mu \mathrm{g} / \mathrm{L})$ & Recuperação (\%) & Média (\%) & $\operatorname{DPR}(\%)$ \\
\hline $1^{\mathrm{o}}$ & Lago Duas Bocas & 1,0 & 90,0 & & \\
\hline $2^{\circ}$ & Lago Duas Bocas & 1,0 & 90,2 & & \\
\hline $3^{\circ}$ & Rio Jucu & 1,0 & 93,6 & & \\
\hline $4^{\circ}$ & Rio Jucu & 1,0 & 94,2 & 94,0 & 3,2 \\
\hline $5^{\circ}$ & Rio Santa Maria & 1,0 & 95,2 & & \\
\hline $6^{\circ}$ & Rio Santa Maria & 1,0 & 96,6 & & \\
\hline $7^{\circ}$ & Rio Santa Maria & 1,0 & 98,0 & & \\
\hline
\end{tabular}

Tabela 4. Valores de recuperação, tempo de retenção, média e coeficiente de variação (CV) para amostras de água do Rio Jucu fortificadas com diferentes concentrações de microcistina-LR $(0,8 ; 1,0$ e $2,0 \mu \mathrm{g} / \mathrm{L})$

\begin{tabular}{|c|c|c|c|c|c|c|}
\hline \multirow{2}{*}{ Mcyst-LR $(\mu \mathrm{g} / \mathrm{L})$} & \multicolumn{3}{|c|}{ Recuperação (\%) } & \multicolumn{3}{|c|}{ Tempo de Retenção (min) } \\
\hline & 0,8 & 1,0 & 2,0 & 0,8 & 1,0 & 2,0 \\
\hline Repetição 01 & 98,2 & 99,5 & 99,2 & 25,754 & 25,802 & 25,879 \\
\hline Repetição 02 & 99,2 & 101,3 & 99,6 & 25,734 & 25,799 & 25,881 \\
\hline Repetição 03 & 98,6 & 101,9 & 104,7 & 25,714 & 25,788 & 25,866 \\
\hline Repetição 04 & 98,5 & 102,2 & 104,8 & 25,698 & 25,724 & 25,769 \\
\hline Repetição 05 & 98,7 & 103,2 & 105,1 & 25,698 & 25,810 & 25,952 \\
\hline Repetição 06 & 99,1 & 104,3 & 105,3 & 25,787 & 25,850 & 25,924 \\
\hline Repetição 07 & 99,2 & 104,9 & 106,1 & 25,770 & 25,829 & 25,920 \\
\hline Média & 98,8 & 102,5 & 103,5 & 25,736 & 25,800 & 25,884 \\
\hline DPR & 0,4 & 1,8 & 2,8 & 0,14 & 0,15 & 0,23 \\
\hline
\end{tabular}

aceitável de recuperação para análise de resíduos está entre 70 e $120 \% .^{21}$ Portanto, a técnica cromatográfica empregando fase móvel à base de metanol aqui validada prova ser eficiente para a análise de Mcyst em amostras ambientais de água.

\section{CONCLUSÃO}

A técnica cromatográfica de CLAE-FR, utilizando $\mathrm{MeOH}$ como modificador orgânico da fase móvel, aqui validada, demonstrou seletividade, linearidade e precisão para separar e quantificar as variantes de Mcyst-RR, Mcyst-YR, Mcyst-LR e Mcyst-LA em diferentes tipologias ambientais de água. A extração em fase sólida, tipo SPE, como tratamento prévio das amostras para a determinação de Mcyst, não alterou a precisão do método, que apresentou níveis de recuperação entre 98,18 e 106,13\%. Os valores de LOD entre $0,17 \mu \mathrm{g} / \mathrm{L}$ (Mcyst-RR) e 0,25 $\mu \mathrm{g} / \mathrm{L}$ (Mcyst-YR) e os valores de LOQ entre $0,55 \mu \mathrm{g} / \mathrm{L}$ (Mcyst-RR) e 0,82 $\mu \mathrm{g} / \mathrm{L}$ (Mcyst-YR) atendem os limites internacionais de potabilidade estabelecida pela Organização Mundial de Saúde. Portanto, o uso do $\mathrm{MeOH}$ se apresenta como uma opção menos tóxica e mais econômica como alternativa a utilização de ACN como modificador orgânico da fase móvel na separação e quantificação de Mcyst por CLAE-FR.

\section{AGRADECIMENTOS}

Os autores agradecem o apoio financeiro recebido da Fundação de Amparo a Pesquisa do Espírito Santo (FAPES) e do Conselho Nacional de Desenvolvimento Científico e Tecnológico (CNPq). Agradecemos também a Universidade Federal do Espírito Santo (UFES) pela disposição de toda a estrutura necessária para o desenvolvimento de experimentos.

\section{REFERÊNCIAS}

1. Carneiro, T.G.; Leite, F.; Rev. Analyt. 2008, 32, 36.

2. Fulweiler, R.W.; Rabalais, N.N.; Heiskanen, A.S.; Mar. Pollut Bull. 2012, 64, 1997.

3. Sivonen, K.; Jones, G.; Em Toxic cyanobacteria in water: a guide to their public health consequences, monitoring and management. Chours, I.; Bartram, J., eds., CRC Press: London, 1999, cap. 3.

4. Yen, H.K.; Lin, T.F.; Liao, P.C.; Toxicon 2011, 58, 209.

5. Sivonen, K.; Appl. Environ. Microbiol. 1990, 56, 2658.

6. Vasconcelos, V.M.; Arch. Hydrobiol. Spec. Issues Advanc. Limn. 1994, $130,439$.

7. Hyenstrand, P.; Blomqvist, P.; Pettersson, A.; Arch. Hydrobiol. Spec. Issues Advanc. Limn. 1998, 51, 41.

8. Parl, H.W.; Hall, N.S.; Calandrino, E.S.; Sci. Total Environ. 2011, 409, 1739.

9. O’Neil, J.M.; Davis, T.W.; Burford, M.A.; Gobler, C.J.; Harmful Algae 2012, 14, 313.

10. Yunes, J.S.; Salomon, P.S.; Matthiensen, A.; Beattie, K.A.; Raggett, S.L.; Codd, G.A.; J. Aquatic. Ecosyst. Health 1996, 5, 223.

11. Matthiensen, A.; Yunes, J.S.; Cood, G.A.; Revista Brasileira de Biologia 1999, $59,1$.

12. Santanna, C.L.; Azevedo, M.T.P.; Nova Hedwigia 2000, 71, 359.

13. Codd, G.A.; Morrison, L.F.; Metcalf, J.S.; Toxicol. Appl. Pharmacol. 2005, 203, 264

14. Hudnell, H.K.; Toxicon. 2010, 55, 1024.

15. Ministério do Meio Ambiente, Conselho Nacional de Meio Ambiente CONAMA. Resolução CONAMA $n^{\circ}$ 357, de 17 março de 2005. Diário Oficial da União. Brasília, seção 1, p.58-63, 2005.

16. Ministério da Saúde. Vigilância e controle da qualidade da água para consumo humano. Brasília, 2006. 
17. Carmichael, W.W.; Sci. Am. 1994, $270,78$.

18. Rapala, J.; Erkomaa, K.; Kukkonrm, K.S.; Lathi, K.; Anal. Chim. Acta. 2002, 466, 213.

19. Mountfirt, D.; Holland, P.; Sprosen, J.; Toxicon 2005, 45, 199.

20. Albuquerque Junior, E.C.; Melo, L.F.C.; Franco, T.T.; Can. J. Anal. Sci. Spectra. 2006, 52, 1 .

21. Purdie, E.L.; Young, F.M.; Menzel, D., Codd, G.A.; Toxicon 2009, 54, 887.

22. Hashimoto, K.; Morimoto, K.; Dobson, S.; Int. Programme Chem. Safety 1993, EHC, 154.

23. Jake L.R.; Siepmanna, J.I.; Schure, M.R.; J. Chromatogr., A. 2011, 1218, 2203.

24. Ribeiro, R. L. V.; Grespan, C. B.; Collins, C. H.; Collins, K. E.; Bruns, R. E.; J. High Resolut. Chromatogr. 1999, 22, 52.

25. Aragão, N.M.; Veloso, M.C.C.; Andrade, J.B.; Quim. Nova 2009, 32, 2476.
26. Ribeiro, R. L. V.; Grespan, C. B.; Collins, C. H.; Collins, K. E.; Bruns, R. E.; J.; J. Braz. Chem. Soc. 2004, 15, 2.

27. Fishbein, L.; Int. Programme Chem. Safety 1997, EHC, 196.

28. Agência Nacional de Vigilância Sanitária - ANVISA. Resolução RE $n^{o}$ 899/2004, de 25 maio de 2003. Diário Oficial da União. Brasília. 2005.

29. Sanches, S.M.; Vieira, E.M.; Prado, E.L.; Benetti, F.; Takayanagui, A.M.M.; Ecl. Quim. 2007, 32, 43.

30. Lawton, L.A.; Edwards, C.; Codd, G.A.; Analyst 1994, 119, 1525.

31. Borges, E.M.; Bottoli, C.B.G.; Collins, C.H.; Quim. Nova 2010, 33, 945.

32. Zhang, L.; Ping, X.; Yang, Z.; Talanta 2004, 62, 193

33. Ribani, M.; Bottoli, C.B.G.; Collins, C.H.; Jardim, I.C.S.F.; Melo, L.F.C.; Quím. Nova 2004, 27, 771.

34. Harada, K.I.; Kondo, F.; Lawton, L.; Em Toxic cyanobacteria in water: a guide to their public health consequences, monitoring and management. Chours, I.; Bartram, J., eds., CRC Press: London, 1999, cap. 3. 